\title{
Aspectos normativos da avaliação das ciências: reflexões para a pesquisa em História das Ciências e da Saúde da Casa de Oswaldo Cruz
}

\author{
Normative aspects of the science evaluation: thoughts for the research \\ in History of the Sciences and Health of the House of Oswaldo Cruz
}

\section{Aspectos normativos de la evaluación de las ciencias: reflexiones para la investigación en Historia de las Ciencias y la Salud de la Casa de Oswaldo Cruz}

Marcus Vinicius Pereira da Silva | marcus.silva@fiocruz.br

Universidade Federal Fluminense. Niterói, Brasil.

Fundação Oswaldo Cruz. Casa de Oswaldo Cruz. Rio de Janeiro, RJ, Brasil.

Lídia Silva de Freitas | lidiasilvadefreitas@gmail.com

Universidade Federal Fluminense. Niterói, Brasil.

Jacqueline Ribeiro Cabral | jacquelinerc@id.uff.br

Universidade Federal Fluminense. Niterói, Brasil.

\section{Resumo}

O trabalho tem como objetivo analisar os aspectos normativos relacionados à avaliação das ciências, na qual se insere a pesquisa em História das Ciências e da Saúde desenvolvida na Casa de Oswaldo Cruz (COC) da Fundação Oswaldo Cruz (Fiocruz). Traz reflexões sobre os principais modelos de avalição das ciências utilizados atualmente, a revisão por pares e indicadores quantitativos, e indica a incorporação de novos modelos, a ciência aberta e os indicadores altmétricos. Verifica-se que todos os modelos são alvo de críticas e que não há consenso sobre um padrão único. Descreve os critérios de avaliação adotados pela COC, pela Fiocruz e pela área de História da Coordenação de Aperfeiçoamento de Pessoal de Nível Superior (Capes). Aponta a criação no âmbito da Fiocruz do Observatório em Ciência, Tecnologia e Inovação e da Política de Acesso Aberto como esforços para superar os limites dos modelos tradicionais de avaliação da ciência e que a área de História da Capes mudou seus critérios de avaliação de periódicos, passando a utilizar a inserção em bases de dados e indicadores bibliométricos na classificação.

Palavras- chave: Gestão de Ciência; Tecnologia e Inovação em Saúde; Recursos para a Pesquisa; Avaliação da Ciência; História das Ciências e da Saúde. 


\begin{abstract}
This paper aims to analyze the normative aspects related to the science evaluation, which is situated the research in History of the Sciences and Health of the House of Oswaldo Cruz (COC) / Oswaldo Cruz Foundation (Fiocruz). Brings reflections on the main models of science evaluation, the peer review and quantitative indicators, and indicates the incorporation of new models, open science and altmetric indicators. Turns out that all models are criticized and there is no consensus on a standard. Describes the evaluation criteria adopted by Fiocruz, COC and the area of History of Coordenação de Aperfeiçoamento de Pessoal de Nivel Superior (Capes). Highlights the creation of the Fiocruz `s Observatory on Science, Technology and Innovation and the Open Access Policy as efforts to overcome the limits of traditional models of science evaluation and the area of History of Capes changed the evaluation criteria for periodicals, beginning to use the insertion in databases and bibliometric indicators in the classification.
\end{abstract}

Keywords: Health Sciences; Technology and Innovation Management; Resources for Research; Science Evaluation; History of the Sciences and Health.

\title{
Resumen
}

El trabajo tiene como objetivo analizar los aspectos normativos relacionados a la evaluación de las ciencias, en que se inserta la investigación en Historia de las Ciencias y de la Salud desarrollada en la Casa de Oswaldo Cruz (COC) de la Fundación Oswaldo Cruz (Fiocruz). Trae reflexiones acerca de los principales modelos de evaluación de la ciencia utilizados actualmente, la revisión por pares y indicadores cuantitativos, y indica la incorporación de nuevos modelos, la ciencia abierta y los indicadores altmetricos. Observa que todos los modelos son objeto de críticas y que no hay consenso sobre un estándar. Describe los criterios de evaluación adoptados por la Fiocruz, la COC y la área de Historia en la Coordenação de Aperfeiçoamento de Pessoal de Nível Superior (Capes). Destaca la creación del Observatorio en Ciencia, Tecnología e Innovación y de la Política de Acceso Abierto de la Fiocruz como esfuerzos para superar los límites de los modelos tradicionales de evaluación de la ciencia y que la área de Historia de la Capes cambió sus criterios de evaluación de periódicos, pasando a utilizar la inserción en bases de datos e indicadores bibliométricos en la clasificación.

Palabras clave: Gestión de Ciencia; Tecnología e Innovación en Salud; Recursos para la Investigación; Evaluación de las Ciencia; Historia de la Ciencia e da la Salud.

\section{Introdução}

Este artigo apresenta parte de uma pesquisa de doutorado em ciência da informação em curso cujo objetivo é analisar a trajetória da pesquisa em História das Ciências e da Saúde (HCS) na Casa de Oswaldo Cruz (COC), unidade técnico-científica da Fundação Oswaldo Cruz (Fiocruz), no que tange à produção e divulgação do conhecimento.

O projeto configura-se como desdobramento de uma ação desenvolvida no âmbito da COC que busca analisar os modos de produção e divulgação do conhecimento técnico e científico da unidade e de seus domínios do conhecimento através de estudos métricos da ciência, de modo que subsidie com informações estratégicas as ações institucionais da unidade. Nesse sentido, os resultados podem ser caracterizados como uma forma de metaconhecimento de orientação nomológica ${ }^{1}$ ao buscar padrões e tendências dos campos científicos por meio de métricas da produção técnica e científica.

Ao longo do desenvolvimento dessa atividade notou-se que apesar da reconhecida importância os estudos quantitativos não respondem a todas as questões colocadas e que devem ser combinadas com estudos qualitativos. Conjectura-se ainda que ocorreram mudanças no processo de produção e divulgação do conhecimento no campo da História das Ciências e da Saúde na COC ao longo dos anos e que há diferentes discursos sobre a prática científica. 
A investigação entende que os critérios de avaliação da ciência no qual o campo analisado está inserido impactam o processo de produção e divulgação do conhecimento. Essas normativas são estabelecidas por diferentes instituições, como as agências de fomento e de regulação, que fazem parte junto com outros sujeitos do campo científico. Nesse cenário, coloca-se a seguinte questão: quais são os contextos normativos nos quais a pesquisa em História das Ciências e da Saúde na COC está inserida?

Diante da pergunta colocada, na primeira parte deste trabalho são apresentados os principais métodos de avaliação da ciência e em seguida alguns quadros normativos nos quais o campo de análise está inserido.

\section{Métodos de avaliação da ciência: a busca por um modelo ideal}

Como ponto de partida para discutir os aspectos normativos na avaliação da ciência são utilizados os estudos desenvolvidos pelo Grupo de Pesquisa “Estudos em Epistemologia, Ética e Política de Informação” (Infoética) liderado pela pesquisadora Maria Nélida Gonzalez de Gomez. A autora lembra que os procedimentos de avaliação das ciências não são recentes ${ }^{2} \mathrm{e}$ ao buscar traçar alguns contextos e condições de constituição desses procedimentos, recorre à obra de Michèle Lamont ${ }^{3}$ e seus estudos em sociologia comparativa da valoração e de Paul W. Taylor ${ }^{4}$ sobre a composição dos discursos normativos e os julgamentos de valor.

Para estudar as práticas valorativas e avaliativas, Lamont ${ }^{3}$ utiliza a categoria de tecnologia do conhecimento desenvolvida por Knorr-Cetina ${ }^{5}$. Segundo Lamont ${ }^{3}$, o trabalho de Knorr-Cetina contribui para tais estudos na medida que propõe analisar os tipos de controles colocados no processo de valoração e avaliação, incluindo o método de comparação, critérios, regras, autoconceitos e outros tipos de elementos não-humanos.

No âmbito da ciência, Lamont ${ }^{3}$ reconhece que, apesar de não estar baseada em preços e mecanismos econômicos, a avaliação pode estar relacionada a premiações e tipos de recompensas que podem ser monetários. Entre as formas de avaliação existentes a revisão por pares (peer review) é uma das mais usadas, especialmente nas ciências categorizadas como humanas e sociais. O método de avaliação das publicações e/ ou projetos pode ser através da classificação, no qual todos os itens são avaliados de acordo com um conjunto de critérios pré-estabelecidos e dois ou mais itens podem ser avaliados com o mesmo grau de qualidade, ou através da elaboração de rankings, no qual os itens são comparados entre eles33.

De acordo com Gonzalez de Gomez ${ }^{2}$, a abordagem de Lamont ${ }^{3}$ é próxima da traçada por Taylor ${ }^{4}$. Este último identifica dois modos diferentes de usar a palavra 'bom': um relacionado a um 'valor qualificativo' (granding) que se aplica "ao domínio da classe universal e às características conceituais de sua demarcação (o que seja ser bom entre todos os passados, atuais e possíveis casos de manifestação de um atributo de valor)”, e a um 'valor hierarquizador' (ranking), "neste caso se aplica a uma classe comparativa situada, na qual o escopo e abrangência dos casos incluídos nas séries ordenadas são estabelecidos por definição" utilizar as categorias de julgamentos de valor propostos por Taylor e Lamont para analisar as tecnologias do conhecimento que formam os sistemas de avaliação da ciência, conforme demonstrado no Quadro 1.

Quadro 1 - Tecnologias do conhecimento: modelos de avaliação científica

\begin{tabular}{|c|c|c|}
\hline Julgamentos de valor & $\begin{array}{l}\text { Revisão por pares } \\
\text { (Peer Review) }\end{array}$ & $\begin{array}{l}\text { Avaliação indireta ou ex-post } \\
\text { (Ex: Análises bibliométricas) }\end{array}$ \\
\hline $\begin{array}{l}\text { Julgamento qualificativo (Valor } \\
\text { de graduação) - TAYLOR; } \\
\text { Estima - LAMONT }\end{array}$ & $\begin{array}{l}\text { Julgamento comparativo a } \\
\text { partir de padrões, matrizes } \\
\text { epistêmicas, paradigmas }\end{array}$ & \\
\hline $\begin{array}{l}\text { Julgamento hierárquico (Valor } \\
\text { de ordenamento) - TAYLOR; } \\
\text { Ranking - LAMONT }\end{array}$ & & $\begin{array}{l}\text { Julgamento comparativo entre os } \\
\text { membros de uma mesma classe (ou } \\
\text { agregado), visando ao ordenamento } \\
\text { hierárquico dos componentes }\end{array}$ \\
\hline
\end{tabular}

Fonte: González de Gomez, 2014. 
Gonzalez de Gomez ${ }^{2}$ cita a revisão por pares, ex-ante, como exemplo de julgamento de valor estimativo ou qualificativo e as que utilizam análises métricas, após a publicação, como exemplo de valor hierarquizador ou sistemas de ranking. Apesar da revisão por pares ser a mais utilizada como instrumento de avaliação da ciência ${ }^{3,6}$, os métodos que utilizam medidas bibliométricas/cientométricas estão se popularizando3.

A revisão por pares é utilizada em diversos contextos, tais como seleção de manuscritos para publicação, premiações, distribuição de recursos e avaliação de grupos de pesquisa e instituições. Entre as críticas, destacam-se: predominância de avaliações diferentes acerca de um mesmo item, avaliações baseadas em critérios não científicos e pouca ligação entre a avaliação e a qualidade dos trabalhos avaliados ${ }^{6,7}$. Outros autores defendem este tipo de avaliação uma vez que ela é realizada somente por especialistas qualificados capazes de julgar apropriadamente a produção de suas áreas. As discordâncias entre os revisores na avaliação podem ainda ser vistas como benéficas, pois ao apresentarem diferentes perspectivas, a confiabilidade diminui, mas a validade do processo aumenta ${ }^{6-8}$. Um dos motivos para a revisão por pares ser o processo mais utilizado seria a inexistência de consenso sobre uma alternativa melhor ${ }^{6,7,9}$.

Nessa perspectiva, Bornmann ${ }^{7}$ propõe que para que o resultado oriundo de uma revisão por pares tenha credibilidade a avaliação deve ser: confiável, ou seja, as contribuições devem obedecer a critérios científicos; ser justa, não favorecendo determinado grupo em detrimento de outro; e ter consistência preditiva, de maneira que os resultados da avaliação devem estar correlacionados com as medidas de desempenho científico subsequentes à decisão.

Diante das críticas a revisão por pares, as análises métricas ganharam espaço nos processos de avaliação das ciências. Essas avaliações nascem como forma de tornar os critérios mais objetivos e confiáveis ${ }^{10}$, mas alguns autores recomendam a conjugação de métodos quantitativos com os qualitativos ${ }^{10,11}$. Entre as vantagens do uso desses indicadores, destacam-se: menor custo e processo mais ágil comparado às avaliações por revisão por pares; reprodutibilidade; acessibilidade internacional e facilidade de interpretação ${ }^{2}$. Não obstante, esses tipos de avaliação também são objetos de diversas críticas que serão pontuadas mais adiante.

Ao problematizar o uso de bases de dados de citações em processos de avaliação das ciências, Freitas ${ }^{12}$ relaciona o uso generalizado de indicadores ao modelo neoliberal de competição internacional, no qual os Estados devem atender a um minimum de desempenho de indicadores sociais quantitativos estabelecidos internacionalmente ${ }^{12,13}$. $\mathrm{O}$ uso de indicadores no processo de avaliação impulsiona os pesquisadores para uma lógica produtivista, pela qual buscam publicar cada vez mais em periódicos considerados de alta qualidade pelas empresas que gerenciam as grandes bases de dados bibliográficas. A autora faz um paralelo dessa lógica com o uso taylorista do controle dos "tempos e movimentos", agora aplicados ao trabalho intelectual de concepção criativa, conforme categorizado por Tauile ${ }^{14}$, e pergunta se "esta seria mais uma investida rumo à sua subordinação real ao capital" ${ }^{\prime 2}$.

Apesar de parecer consenso entre os pesquisadores que a avaliação da ciência é importante e necessária dado a necessidade de alocação de recursos de pesquisa e premiações, não há quando a discussão é acerca do método a ser empregado. Para exemplificar, apresenta-se algumas falas do debate promovido pelo periódico "Cadernos de Saúde Pública" publicado em 2013.

O artigo que abre a seção para debate de autoria de Camargo Jr. ${ }^{15}$ traz uma perspectiva crítica ao uso de indicadores quantitativos para avaliar a ciência. Para o autor, não existe métrica adequada para mensurar a qualidade de uma pesquisa e a aparente objetividade desses indicadores faz com que não se questione $o$ aspecto fundamental da utilização, seu sentido. $O$ uso excessivo traria consequências prejudiciais para a ciência, como a preferência dos pesquisadores para publicação de apenas resultados positivos de pesquisa, alto número de retratações por má conduta da pesquisa, a prática do salami science, coautorias de pesquisadores que não contribuíram efetivamente na pesquisa, citações indevidas, e redução da possibilidade de inovação científica. Questiona que, se ao depender de ferramentas algorítmicas para avaliar uma pesquisa, os avaliadores não estariam sendo "covardes intelectuais" e propõe como alternativa um sistema baseado 
na revisão por pares, no qual os autores indicariam seus 5 melhores trabalhos para serem avaliados qualitativamente.

Nísia Trindade Lima ${ }^{16}$, atual presidente da Fiocruz e pesquisadora do campo das ciências humanas e sociais na saúde, acrescenta outros prejuízos advindos do uso de indicadores quantitativos, sendo eles: desvalorização da publicação de livros, desconsideração de atividades importantes para o desenvolvimento da ciência e sobrecarga de trabalho para os pesquisadores. Em relação ao sistema de avaliação da Capes, sinaliza que, se por um lado possibilitou importantes avanços na pesquisa e ensino, por outro dificultou o trabalho interdisciplinar entre as diferentes áreas do conhecimento da Fiocruz, posto que não existe classificação única dos periódicos para as diferentes áreas. Por fim, aponta a necessidade de aprofundar os debates e indicar novos parâmetros para a avaliação da ciência.

Maurício Barreto ${ }^{17}$ também tece críticas ao uso dos indicadores bibliométricos devido às suas deficiências metodológicas e de validação. Assim como Lima $^{16}$, destaca que esses indicadores excluem processos importantes da pesquisa. Ressalta que atualmente há poucas alternativas e que não tem certeza se o melhor caminho seria a adoção das avaliações qualitativas.

Em contraponto, outros pesquisadores questionam se o uso exclusivo de indicadores qualitativos é o melhor caminho ${ }^{18-20}$. Struchiner ${ }^{20}$ lembra que os comitês assessores do CNPqjá se basearam em metodologias qualitativas e o resultado não foi positivo. Problemas em relação à parcialidade dos pareceristas também são apontados ${ }^{18-20}$.

Recentemente observa-se esforços em melhorar os sistemas de avaliação da pesquisa baseados em indicadores quantitativos, como o Leiden Manifesto for research metrics ${ }^{21}$. Esse documento apresenta dez princípios para uma avaliação da pesquisa baseada em métricas de modo que os pesquisadores possam confiar na avaliação e os avaliadores possam confiar nos seus indicadores, sendo eles: 1) avaliação quantitativa deve dar suporte à avaliação qualitativa especializada; 2) medir o desempenho de acordo com a missão da instituição, do grupo ou do pesquisador; 3) proteger a excelência da pesquisa localmente relevante; 4) manter a coleta de dados e os processos analíticos abertos, transparentes e simples; 5) permitir que os avaliados verifiquem os dados e as análises; 6) considerar as diferenças entre áreas nas práticas de publicação e citação; 7) basear a avaliação de pesquisadores individuais no juízo qualitativo da sua carreira; 8) evitar rigidez.

Diante das diversas críticas aos principais métodos de avaliação da ciência, revisão por pares e indicadores bibliométricos, emergem alternativas que estão ganhando visibilidade nos últimos anos. Apresenta-se, neste trabalho, duas possibilidades de avaliação alternativa, uma relacionada ao movimento de ciência aberta e outra ao uso de métricas alternativas (altmetria), como apresentado no Force11 Manifesto.

De acordo com Freitas ${ }^{12}$, o Force 11 Manisfesto tem como objetivo implementar métricas que atendam um maior número de atividades das ciências, dado que os indicadores tradicionais negligenciam muitas delas, como as atividades de orientação, o reconhecimento pelos pares e as citações na mídia do chamado 'intelectual público'.

Entre as métricas alternativas, a altmetria se destaca. E pode ser entendida como a forma de medir a ciência baseada em indicadores produzidos a partir de ferramentas e ambientes online, como blogs, Wikipédia, Facebook, Twitter, ResearchGate. Segundo Vanti e Sanz-Casado ${ }^{23}$, sua criação deve-se à insatisfação com os indicadores tradicionais, ao surgimento das novas ferramentas, à necessidade de novos filtros para selecionar informação relevante na ciência e ao movimento de open access.

Ao comparar o uso de indicadores altmétricos com o indicador bibliométrico índice-h, Gouveia ${ }^{24}$ frisa que nos primeiros os dados utilizados são potencialmente mais manipuláveis, porém a manipulação é facilmente detectável. Ao utilizar ferramentas que não são restritas ao meio acadêmico eles podem ser empregados para medir o impacto social da ciência e contribuir para a avaliação da ciência ao passo que complementam os indicadores tradicionais. Questiona-se, no entanto, se a repercussão de trabalhos científicos em mídias 
sociais não estaria relacionada com uma midiatização da ciência. Na medida em que esses indicadores passam a ser incorporados pelas agências de fomento e instituições de pesquisa para avaliar as ciências, os temas de pesquisa que não possuem apelo social e midiático não seriam prejudicados?

No tocante à ciência aberta é possível dizer que ela advém do movimento de acesso aberto que nasce de forma organizada no início da década de 2000 com a publicação da Declaração de Budapeste ${ }^{22}$. A ciência aberta, à medida que permite que a própria comunidade científica verifique a qualidade das pesquisas, enfraquece o poder dos pareceristas do sistema de revisão por pares e torna consequentemente as avaliações abertas e mais transparentes.

Afinal, verifica-se que não há consenso entre os pesquisadores e gestores quanto ao melhor método para avaliar a ciência. Indaga-se, no entanto, se é possível alcançá-lo.

\section{A avaliação da pesquisa no âmbito do campo da História das Ciências e da Saúde na COC}

A Fiocruz, instituição no qual a COC está inserida, possui diversas áreas de atuação: ensino (médio técnico, pós-graduação Lato-sensu e Stricto-sensu), produção de fármacos e vacinas, serviços de saúde, pesquisa, vigilância em saúde, entre outros. No que se refere à pesquisa, a Fiocruz possui 29 áreas, atuando desde em áreas caracterizadas como ciências duras às áreas conhecidas como ciências humanas e sociais ${ }^{25}$.

Diante da diversidade de linhas de ação em uma área sensível à sociedade e de grande interesse político e econômico, a saúde, a pesquisa em História das Ciências e Saúde desenvolvida na COC enfrenta dentro da Fiocruz um campo de disputas pelo reconhecimento, legitimação e captação de recursos.

Ao estar inserida na Fiocruz e a maior parte dos seus pesquisadores fazer parte do corpo docente do Programa de Pós-Graduação em História das Ciências (PPGHCS), a pesquisa em História das Ciências e da Saúde da COC tem como principais sistemas de avaliação: o a da própria unidade, da Fiocruz e da área de História na Capes.

O PPGHCS foi criado em 2001, 15 anos após a COC. Em entrevista à Castro ${ }^{26}$, alguns pesquisadores da unidade apontam que o modo de funcionamento da pesquisa foi alterado em decorrência da criação do Programa e sua subordinação aos critérios de avaliação da Coordenação de Aperfeiçoamento de Pessoal de Nível Superior (Capes).

Sobre a criação do programa de pós-graduação, um pesquisador aponta que naquele momento não havia entendimento entre os membros da academia se institutos de pesquisa deveriam ter uma área ensino. Segundo o pesquisador,

"muitas pessoas diziam: não, não deve ter ensino, ensino tem que estar na faculdade, esses lugares devem ser só pra pesquisa. Essa ideia, durante muito tempo, foi hegemônica. [...] O Ensino era lá na faculdade, pras pessoas que podem gastar mais tempo ensinando. A gente vai fazer pesquisa. Vai ser produtivo em pesquisa porque não precisamos gastar nosso tempo dando aula, orientando. Esse processo foi mudado nas financiadoras, na política científica brasileira. A pesquisa tem que se retroalimentar na pós-graduação. Tanto pra melhorar o ensino, como pra ampliar o escopo da pesquisa. Então, assim, isso mudou depois. Aí a pós surgiu e cada vez mais a pós é importante. Mas isso não é uma dinâmica da Casa. É uma dinâmica da pesquisa brasileira” (Pesquisador 1).

Esta fala diverge de outro depoente que atuou diversas vezes em cargos de gestão na unidade. Ele aponta que em determinado momento a $\mathrm{COC}$ incentivou seus pesquisadores a fazerem doutorado com o objetivo de criar um programa de pós-graduação e destaca que a ação da unidade não foi isolada, ela está relacionada à ampliação dos cursos de pós-graduação no nível nacional, o reconhecimento do plano de carreira no 
qual o título de mestre e doutor converge em gratificação financeira e o incentivo da Fiocruz. Segundo o entrevistado,

"Na hora que você sinaliza que todo mundo tem que fazer o doutorado, já é com a perspectiva que no futuro a gente vai criar uma pós-graduação. Isso estava no horizonte. Tanto que aí, depois que todo mundo se graduou, quer dizer, quando a maioria dos pesquisadores concluiu seus doutorados, a gente já estava pronto pra começar a abrir um programa de pós-graduação. Mas isso tudo é uma estratégia. Uma estratégia institucional" (Pesquisador 2).

Apesar da divergência sobre a intencionalidade original de se criar um programa de pós-graduação na COC, ambos afirmam que a criação alterou de maneira brusca o Departamento de Pesquisa em História das Ciências e da Saúde (Depes) e a pesquisa desenvolvida no Depes passa a ser confundida com o PPGHCS e vice-versa. Destacam que a pós-graduação nasce como um grande projeto e acaba absorvendo as ações desenvolvidas no Depes.

Um dos entrevistados critica ainda os modelos avaliativos das agências reguladoras de pós-graduação no Brasil, no qual a exigência de produtividade cerceia a criatividade e a produção em outras áreas do conhecimento. De acordo com o pesquisador,

"Ao mesmo tempo em que a pós-graduação é uma coisa fabulosa, assim, de contato com o aluno, $e$ renovação, cada vez mais as instituições que coordenam a pós-graduação no Brasil, Capes, CNPq, faz com que a pós-graduação seja uma coisa cada vez mais presa a ditames, assim, totalmente sem sentido. [...] Cada vez mais trabalhando mais também, se a gente antigamente tinha um trabalho tranquilo, de vir aqui, escrever, e pesquisar fontes, cada vez mais é preparar aula, é fazer coisas pra orientar e o trabalho ampliando. [...] Pra escrever um artigo, a gente pensa dez vezes aonde vai botar, com quem vai escrever, porque... não é de acordo com as nossas preferências. Essa é a parte chata, que eu falei pra você, dentro do nosso trabalho. Cada vez mais ela é formalizada" (Pesquisador 1).

O PPGHCS é avaliado pela área de História da Capes. Apesar ter como principal objetivo a avaliação dos programas de pós-graduação Stricto-sensu brasileiros, ela influencia as pesquisas em todo país, dado que a maior parte é desenvolvida no âmbito da pós-graduação e as classificações dos periódicos (Qualis-Periódi$\operatorname{cos)}$ são usadas inadequadamente para outros fins.

Entre os critérios de avaliação adotados pelas áreas, um dedica-se à avaliação da produção intelectual dos docentes e discentes. Cada área tem autonomia para atribuir peso para produção intelectual e definir os tipos de documentos que são avaliados. Na área de História o critério da avaliação quadrienal 20132016 estipula o peso de 40\% para a produção intelectual, desses: 55\% referem-se à publicação de artigos, capítulos de livros e livros, $30 \%$ à distribuição das publicações por docentes e $15 \%$ à produção técnica.

Em relação aos livros, a área de História utiliza atualmente como tecnologia do conhecimento de avaliação científica ${ }^{3}$ a revisão por pares feita por uma comissão de pesquisadores indicados pelo coordenador da área e eles são classificados em 4 diferentes notas: L1, L2, L3 e L4, sendo essa última a nota máxima. Sendo assim, a área realiza um julgamento de valor qualificativo aos livros.

No caso dos periódicos, a área adota um conjunto de critérios quantitativos e qualitativos -atribui tanto um julgamento de valor qualificativo quanto hierárquico. Assim como os livros, a avaliação também é feita por uma comissão de pesquisadores indicados pelo coordenador da área.

A área mudou os critérios de avaliação de periódicos ao longo dos anos. Até 2006 todas as áreas de avaliação da Capes separavam os periódicos segundo a circulação (internacional, nacional ou local) e em cada grupo as revistas eram classificadas nos estratos A, B ou C, sendo A o estrato mais alto ${ }^{27}$. Após o 
diagnóstico do uso inapropriado da classificação e intensa discussão, foi aprovada em 2007 uma nova classificação contendo sete estratos: A1, A2, B1, B2, B3, B4, B5 e C, no qual A1 é o mais alto e C é usado para as publicações que não consideradas periódicos científicos ou não atendam aos critérios mínimos estabelecidos pela área ${ }^{27}$.

$\mathrm{Na}$ avaliação relativa aos anos 2007-2009 a História considerava a presença dos periódicos nas bases de dados e indexadores para a classificação como o SciELO, Scopus, PsycInfo e ISI, porém não considerava o fator de impacto. O documento de área 2009 reconhece que as publicações no campo não estão indexadas nas grandes bases internacionais. Frisa que a classificação dos periódicos científicos exclui outra importante produção acadêmica relevante para a área - as publicações em revistas e jornais de divulgação científica - e que a área tem feito o esforço de inserir seus periódicos nos indexadores e bases de dados nacionais e internacionais ${ }^{28}$.

Ainda na avaliação dos anos 2010-2012 a área não utilizou o fator de impacto e a inserção dos periódicos em bases de dados justificando que poucos periódicos da área eram indexados pelas bases responsáveis pelo cálculo. Por conseguinte, a área adotou critérios qualitativos, segundo eles universalmente valorizados pela comunidade científica em geral ${ }^{29}$.

Já na avaliação 2013-2016 a área voltou a usar a inserção dos periódicos nas bases de dados como critério para classificá-los nos estratos superiores (A1, A2 e B1) e passou a usar os indicadores bibliométricos JCR, SJR e, especialmente, o índice H fornecido pelo Google Scholar de modo suplementar. Segundo o documento de área, a maioria dos periódicos tradicionalmente classificados nos maiores estratos já atendia esses requisitos. Desse modo, a avaliação se tornou mais objetiva. A adoção desses novos critérios serviu então para diferenciar os periódicos dos estratos inferiores e afirmam que a inserção em uma ou mais bases de dados qualifica o periódico. Ao incorporar esses novos critérios, a História se aproxima dos modos de avaliação tradicionais das ditas ciências duras. Como os pesquisadores da área costumam publicar em revistas de outros campos, a avaliação buscou a aproximação com o estrato atribuído pela área de referência. Utiliza também o julgamento qualificativo segundo alguns critérios pré-estabelecidos pela área ${ }^{30}$.

No âmbito da Fiocruz, os indicadores globais (válidos para toda Fiocruz) e as metas que foram utilizadas para a Avaliação do Desempenho Institucional do ano de 2016 privilegiaram os métodos quantitativos. O indicador global relacionado à produtividade em pesquisa é restrito ao número de artigos publicados em periódicos indexados. Segundo o documento, o indicador possibilita a comparabilidade da Fiocruz às outras instituições de ensino superior, sendo considerado um indicador clássico para avaliação da atividade científica $^{31}$. Além disso, a Fiocruz usa outro indicador quantitativo para medir o crescimento real da produção científica através da publicação de artigos em periódicos indexados. Nesse contexto, as ciências humanas e sociais podem ser prejudicadas visto que tradicionalmente privilegiam a publicação em livros e capítulos de livros em detrimento de artigos publicados em periódicos indexados, apesar do recente crescimento do número de publicações em artigos científicos ${ }^{31}$.

Em relação aos indicadores intermediários (específicos para cada unidade), não há padrão acerca dos tipos de publicação contabilizados como metas de produtividade em pesquisa. Das 14 unidades ou escritórios que estabeleceram essas metas, metade considera somente artigos publicados em periódicos indexados. Dessas, duas unidades, o Instituto Oswaldo Cruz (IOC) e o Instituto Nacional de Infectologia (INI), também estipulam metas relacionadas ao fator de impacto.

A Casa de Oswaldo Cruz e outras 6 unidades/escritórios consideram outros tipos de publicação como produtividade em pesquisa. Sendo que a $\mathrm{COC}$ contabiliza artigos publicados em periódicos indexados, livros autorais, capítulo de livro e coletâneas organizadas. Algumas unidades/escritórios não fazem distinção entre periódicos indexados ou não, consideram publicação em sítios na Web e somente uma considera a produção técnica. 
A partir do questionamento do modelo predominante de avaliação da pesquisa marcado pela lógica produtivista, pela priorização de indicadores quantitativos e pelo não reconhecimento das diferenças entre as áreas do conhecimento, a Fiocruz lançou em 2016 o Observatório em Ciência, Tecnologia e Inovação em Saúde da Fiocruz com objetivo de contribuir para a gestão e formulação de políticas institucionais de Ciência, Tecnologia e Inovação baseadas em evidências científicas (indicadores, métricas e análises quantiqualitativas) e gerar informações sobre o impacto social do conhecimento produzido pela instituição ${ }^{32}$.

Ainda que em estágio inicial, o Observatório impulsionou discussões sobre a avaliação da ciência no âmbito da instituição dado que o processo de definição de escopo e de indicadores contou com a participação de pesquisadores e gestores de diferentes áreas do conhecimento da Fiocruz e de consultores externos. Ao buscar realizar estudos quali-quanti, ela vai ao encontro das proposições de alguns autores apontadas na seção anterior deste trabalho.

Outro fator relacionado à avaliação da pesquisa no âmbito da Fiocruz é a instalação da Política de Acesso Aberto ao Conhecimento em 2014. Essa política possui como um dos objetivos, contribuir para o aumento do acesso e impacto da produção intelectual da Fiocruz e promove, como caráter mandatório, o depósito de todas as teses e dissertações dos programas de pós-graduação e dos artigos publicados pelos pesquisadores no repositório institucional da Fiocruz. O cumprimento do depósito propiciará a atribuição de vantagens aos autores na concorrência de editais internos ou de outros mecanismos de recursos próprios da Fiocruz ${ }^{33}$.

\section{Considerações finais}

Este trabalho, ainda que em estágio inicial, possibilitou compreender alguns fatores que estão relacionados à trajetória da pesquisa em História das Ciências e da Saúde da Casa de Oswaldo Cruz e fatores que impactam nos seus processos de produção e divulgação do conhecimento.

Foi possível perceber que os dois principais modelos de avaliação da ciência são alvo de diversas críticas da comunidade científica, assim como os métodos alternativos criados com o objetivo de melhorar os sistemas de avaliação. Por conseguinte, verifica-se que ainda não existe um modelo ideal e chega a se perguntar se é possível existir.

A pesquisa em História das Ciências e da Saúde está inserida em diferentes quadros normativos relacionados entre si, destacando-se o sistema de avaliação da Fiocruz, da própria unidade e da área de História da Capes.

A Fiocruz é reconhecida como uma instituição estratégica para o Estado, especialmente devido as suas áreas de produção de fármacos e vacinas, de pesquisas biomédicas e epidemiológicas e vigilância em saúde. Contudo a instituição possui outras áreas de atuação e campos científicos com práticas científicas distintas, dificultando a avaliação da pesquisa como um todo. Ao criar o Observatório em Ciência, Tecnologia e Inovação em Saúde, a instituição se esforça para criar indicadores que respeitam as especificidades das suas múltiplas áreas e reconhece que os indicadores tradicionais usados possuem limitações.

Em respeito aos critérios adotados pela área de História na Capes, nota-se que eles foram alterados nas três últimas avaliações. Essas mudanças podem ser ocasionadas por divergências dentro da própria área de História sobre qual o melhor critério.

Por fim, reconhece-se que para compreender os impactos que os modos de avaliação expostos acima causam no processo de produção e circulação do conhecimento no âmbito da pesquisa em História das Ciências e da Saúde na COC é necessário confrontá-los com estudos quantitativos para verificar as possíveis mudanças do perfil da produção intelectual dos pesquisadores e qualitativos para investigar suas percepções sobre o tema. 


\section{Referências}

1. González de Gómez MN. Para uma reflexão epistemológica acerca da Ciência da Informação. Perspectivas em Ciência da Informação. 2001;6(1): 5-18.

2. González de Gómez MM. Da questão da validade ao julgamento de valor: mediação informacional da avaliação científica. In: Freire IM, et al., organizadores. Além das nuvens: expandindo as fronteiras da ciência da informação. Anais do XV Encontro Nacional de Pesquisa em Ciência da Informação; 2014; Belo Horizonte. Belo Horizonte: UFMG; 2014. p. 216-36.

3. Lamont M. Toward a comparative sociology of valuation and evaluation. Annual Review of Sociolog. 2012;38(1): 201-21.

4. Taylor PW. Normative Discourse. New York: Prentice-Hall; 1961.

5. Knorr-Cetina K. Epistemic Cultures: how the sciences make knowledge. Harvard University Press; 1999.

6. 7. Bornmann L. Scientific peer review: an analysis of the peer review process from the perspective of Sociology of Science theories. Human Architecture. 2008;6(2):23-38.

7. Bornmann L. Scientific peer review. Annual review of information science and technology. 2011;45(1):197-245.

8. Marsh HW, Ball S. Reflections on the peer review process. Behavioral and Brain Sciences. $1991 ; 14(1): 157-58$.

9. Young SN. Peer review of manuscripts: theory and practice. Journal of Psychiatry \& Neuroscience. 2003;28(5):327-30.

10. Vanti N. Métodos cuantitativos de evaluación de la ciencia. Investigación Bibliotecológica. 2000;14(29):9-23.

11. Mugnaini R, Digiampietri LA, Mena-Chalco J. Comunicação científica no Brasil (1998-2012): indexação, crescimento, fluxo e dispersão. Transinformação. 2014;26(3):239-52.

12. Freitas LS. Bases de dados e indicadores na produção científica: articulando informação, conhecimento e ética. In: González de Gómez MM, Cianconi RB, organizadoras. Ética da informação: desafios e perspectivas. Rio de Janeiro: Garamond; 2017. p. 110-29.

13. Senra NC. Informação estatística como objeto de estudo: uma primeira tentativa de formalização. Datagramazero. 2005;6(4).

14. Tauile JR. Uma introdução à Economia Política da informação. Ensaios FEE. 1981;2(2):89-108.

15. Camargo JR. KR. Produção científica: avaliação da qualidade ou ficção contábil? Cadernos de Saúde Pública. 2013;29(9):1707-11.

16. Lima NT. Avaliação desmedida. Cadernos de Saúde Pública. 2013;29(9):1723-25.

17. Barreto ML. Como avaliar as ciências com uma deficiente ciência da avaliação científica? Cadernos de Saúde Pública. 2013;29(9):1719-21.

18. Vaz P. Por um novo jogo. Cadernos de Saúde Pública. 2013;29(9):1713-14.

19. Barata RB. Medir ou classificar a produção científica de pesquisadores? Cadernos de Saúde Pública. 2013;29(9):1712-13.

20. Struchiner $\mathrm{CJ}$. Avaliação da qualidade da produção científica e suas consequências imprevistas e indesejadas: um conceito autoevidente? Cadernos de Saúde Pública. 2013;29(9): 1716-17.

21. Hicks D, et al. Bibliometrics: The Leiden Manifesto for research metrics. Nature. 2015;502(7548):429-31.

22. Gomes SLR. O Acesso Aberto ao conhecimento científico: o papel da universidade brasileira. RECIIS. 2014;8(2):93-106.

23. Vanti N, Sanz-Casado E. Altmetria: a métrica social a serviço de uma ciência mais democrática. Transinformação. 2016;28(3):349-58.

24. Gouveia FC. Impacto Real e Imediato? [Internet]. Rio de Janeiro: Fiocruz; 2016 [citado em 5 set. 2017]. Disponível em: http://observatorio.fiocruz.br/ponto-de-vista/altmetria. 
25. Fundação Oswaldo Cruz. Áreas de pesquisa [Internet]. Rio de Janeiro: Fiocruz; 2014 [acesso em 10 set. 2017]. Disponível em: https://portal.fiocruz.br/pt-br/content/grandes-áreas-temáticas.

26. Castro EL. Conhecimento e memória na Casa de Oswaldo Cruz/Fiocruz: reflexões e elementos para a construção de iniciativas de memória organizacional [dissertação]. Rio de Janeiro: Instituto Brasileiro de Informação em Ciência e Tecnológica; 2016. 190 f.

27. Barata RCB. Dez coisas que você deveria saber sobre o Qualis. RBPG. 2016;13(30):13-40.

28. Coordenação de Aperfeiçoamento de Pessoal de Nível Superior. Documento de área 2013: História. Brasília, DF: Capes; 2009.

29. Coordenação de Aperfeiçoamento de Pessoal de Nível Superior. Documento de área 2013: História. Brasília, DF: Capes; 2013.

30. Coordenação de Aperfeiçoamento de Pessoal de Nível Superior. Documento de área: História. Brasília, DF: Capes; 2016.

31. Rego TC. Produtivismo, pesquisa e comunicação científica: entre o veneno e o remédio. Educação e Pesquisa. 2014;40(2):325-46.

32. Fundação Oswaldo Cruz. Observatório em Ciência, Tecnologia e Inovação em Saúde da Fiocruz: sumário executivo [Internet]. Rio de Janeiro: Fiocruz; [citado em 10 Set 2016]. Disponível em: http:// observatorio.fiocruz.br/sites/default/files/sumario bilingue observatorio.pdf.

33. Fundação Oswaldo Cruz. Portaria da presidência n 329/2014. Rio de Janeiro: Fiocruz; 2014. 